\title{
The Association of Anxiety Severity With Health Risk Behaviors in a Large Representative Sample of Korean Adolescents
}

\author{
Kyung Soo Woo, Yoonmi Ji, Hye Jeong Lee, and Tae Young Choi \\ Department of Psychiatry, Daegu Catholic University School of Medicine, Daegu, Korea
}

\begin{abstract}
Objectives: Anxiety disorders are the most common psychiatric disorders in adolescents and seem to occur the earliest among all forms of psychopathology. The aim of this study was to investigate the association of anxiety severity with health risk behaviors and mental health in adolescents.

Methods: Data from the 2020 Korean Youth Risk Behavior Web-Based Survey were analyzed. A total of 54948 adolescents responded to the 7-item Generalized Anxiety Disorder Scale (GAD-7) for the assessment of their anxiety severity as well as to the mental health and health risk behavior survey. Logistic regression analysis, $t$ tests, and variance analysis of a complex sample general linear model were used to examine the association of anxiety severity with health behaviors and mental health.

Results: After statistical adjustment for sociodemographic characteristics, the subjects in the severe anxiety group were significantly more likely to be current smokers (odds ratio [OR]: 2.08, 95\% confidence interval [CI]: 1.72-2.50), current drinkers (OR: 1.91, 95\% CI: 1.67-2.19), experience habitual substance use (OR: 10.89, 95\% CI: 8.22-14.42), have sexual intercourse (OR: 2.10, 95\% CI: 1.76-2.51), and have unprotected intercourse (OR: $2.21,95 \%$ CI: 1.67-2.92) than those in the normal group. Anxiety severity negatively correlated with sleep satisfaction and happiness, but positively correlated with stress perception, loneliness, depressive symptoms, and suicidality. Conclusion: Adolescent anxiety is associated with health risk behaviors and poor mental health. Thus, early screening and intervention for anxiety in adolescents could contribute to the management and coping of youth health risk behaviors in the community.
\end{abstract}

Keywords: Adolescents; Anxiety; Health risk behaviors; Generalized Anxiety Disorder Scale; Mental health.

Received: June 30, 2021 / Revision: July 23, 2021 / Accepted: July 26, 2021

Address for correspondence: Tae Young Choi, Department of Psychiatry, Daegu Catholic University School of Medicine, 33 Duryugongwon-ro 17-gil, Nam-gu, Daegu 42472, Korea

Tel: +82-53-650-4786, Fax: +82-53-623-7507, E-mail: tyoungchoi@cu.ac.kr

\section{INTRODUCTION}

Anxiety is a normal biological response that arises upon feeling threatened. However, it can develop pathological features if the symptoms persist and daily functioning is impaired. Anxiety disorders are characterized by excessive and persistent fear and worry that interrupt daily life, cause significant distress [1], and may be accompanied by physical symptoms, such as abdominal distress, headache, and increased heart rate.

Anxiety disorders are the most common mental disorders in children and adolescents, and anxiety disorders in childhood and adolescence may be the first manifestation of a variety psychopathologies [2]. Childhood and adolescence is a vulnerable period for the occurrence of anxiety symptoms, which widely range from mild temporary symptoms to an

This is an Open Access article distributed under the terms of the Creative Commons Attribution Non-Commercial License (https://creativecommons.org/licenses/by-nc/4.0) which permits unrestricted non-commercial use, distribution, and reproduction in any medium, provided the original work is properly cited. anxiety disorder that meets the full diagnostic criteria. According to a US nationwide survey conducted with 10123 adolescents aged 13-18 years, the prevalence of all types of anxiety disorder was $31.9 \%$, making it the most common mental disorder in this population [3]. In another study, it was observed that anxiety disorder occurring in adolescence became chronic, persisting into adulthood or commonly cooccurring with other mental disorders, like depression [4]. In a longitudinal study with 1228 adolescents, $20 \%$ of those who met the full diagnostic criteria for any anxiety disorder (panic disorder, agoraphobia, specific phobia, social phobia, generalized anxiety disorder) still met the criteria at the 19 months follow-up, and $42 \%$ of the adolescents who did not meet the diagnostic criteria for an anxiety disorder were still experiencing anxiety symptoms at the follow-up, following a chronic course [5]. The National Survey of Children's Health conducted in the US in 2016 reported that approximately 4.4 million children and adolescents aged 3-17 years (7.1\% of this age group in the US) were diagnosed with an 
anxiety disorder, and that about one in three adolescents with anxiety disorder showed conduct problems (37.9\%) and depression (32.3\%). In addition, drug and alcohol abuse or dependence were found to commonly co-occur with anxiety disorder [6].

Anxiety disorders in childhood and adolescence are underdiagnosed or frequently untreated even if diagnosed. Hence, aggressive intervention is often needed. Children can express fear and worry during the normal developmental process; thus, it can be difficult to differentiate this process from pathological anxiety. Children and adolescents may seem to seriously suffer from normal anxiety, but most of the time it is a transient phenomenon [7]. Accordingly, in children, the severity of distress due to anxiety is not an appropriate criterion with which to differentiate normal and pathological anxiety. Anxiety symptoms in children and adolescents may have different manifestations than those in adults. However, in the current diagnostic system, the same diagnostic criteria for anxiety disorder are used in adults as well as in children and adolescents, with the exception of separation anxiety disorder, and the clinical characteristics (e.g., duration, type of symptoms, frequency) unique to children and adolescents are mentioned only in a few anxiety disorders [1]. Because children and adolescents may have difficulties in communicating cognition, language, and emotions due to developmental differences, differences in cognitive development should be considered in the diagnostic evaluation of and approach to anxiety. However, there are no such guidelines in the current diagnostic system. Moreover, anxiety disorders are the most untreated mental problem in adolescence and currently, only $18 \%$ of adolescents with any anxiety disorders are under treatment [8].

On the other hand, health risk behaviors, which are behaviors resulting in negative health outcomes (such as substance use, precocious sexual experience, unprotected intercourse, and violence), increase during adolescence [9]. Health risk behaviors do not occur in isolation, but tend to co-occur with other risk behaviors. The two types of risk behavior are mutually predictive because they share similar risk factors [10]. The earlier the health risk behaviors occur, the more likely it is to lead to substance addiction, substance abuse, physical and mental health worsening, and occupational and educational underachievement during overall adolescence and adulthood [11]. Accordingly, many countries pay close attention to health risk behaviors as a key to public health policy. Generally, anxiety is known to be associated with decision-making to avoid risk. However, evidence has steadily accumulated that excessive anxiety is linked to smoking, drinking, substance abuse, unprotected sexual intercourse, gambling, and other unhealthy habits, and that health risk behavior may worsen as a means to control anxiety [12].

Previous studies emphasized the importance of early assessment and the intervention of anxiety in adolescence. However, few studies in Korea to date reported the relationship of anxiety symptoms to mental health and health risk behavior based on a large sample.

Currently, the Korea Disease Control and Prevention Agency (KDCA) conducts the Korea Youth Risk Behavior Webbased Survey (KYRBS) on large representative samples of 7th-12th graders every year to examine adolescents' health behaviors. We hypothesized that anxiety, which may be an early stage of various psychopathologies, is associated with other mental health conditions and health risk behaviors of Korean adolescents. To evaluate our hypothesis, we first identified the sociodemographic characteristics of communitybased adolescents suffering from anxiety by classifying anxiety severity. Our ultimate objective was to present valuable findings to promote and manage the mental health of adolescents with anxiety by shedding light on the association between anxiety severity and mental health and health risk behaviors in these adolescents.

\section{METHODS}

\section{Participants}

The raw data of the 16th KYRBS, conducted from August 3 to November 13, 2020 and provided by the KDCA, were analyzed. The annual survey has been performed by the KDCA since 2005 to examine Korean adolescents' health behaviors, including smoking, drinking, and physical activities. Each year, the survey is administered to the students of 400 middle schools and 400 high schools sampled in the 17 first-tier administrative divisions nationwide, with the exceptions of chronic absentees, special education students, and students with dyslexia. The raw data of the 16th wave survey were the first data to contain scores of the 7-item Generalized Anxiety Disorder Scale (GAD-7), and were used in this study to evaluate anxiety levels. Students anonymously selfreported the survey with 103 items across 15 domains by connecting to the homepage in a group setting at school computer labs or by utilizing mobile devices under faculty supervision in classrooms if computer labs were unavailable due to coronavirus disease. It took 45-50 $\mathrm{min}$ to fill out the survey. A total of 57925 students were selected. Of those, 54948 (94.9\%) participated in the survey. As this study was conducted by utilizing raw data of a government-approved statistical survey (approval no. 117058), the study was exempted from an Institutional Review Board review (CR-21-086). 


\section{Measurement variables}

\section{Sociodemographic variables}

Sociodemographic variables included age, sex, family affluence scale, area of residence, academic achievement, and history of violence victimization. The family affluence scale was developed to measure the absolute level of economic status and consisted of items that were easy for adolescents to self-report. The scale showed a high level of agreement between adolescents and parents [13]. In the 16th wave survey, items regarding the number of cars at home, whether the student had his/her own room, the number of overseas travels, the number of computers at home, the number of bathrooms at home, and whether the household had an air purifier were used in the family affluence scale. The scale was scored between 0 and 13 points. The area of residence was classified into rural area, small-to-medium city, and large city. With regards to academic achievement, students were asked about their grades in the last 12 months and were instructed to select one of the following answer choices: high, high-middle, middle, low-middle, and low. With regards to the history of violence victimization, the presence of a history was determined if students responded "once or more" to the question, "In the last 12 months, have you been treated at the hospital due to violence from friends, senior students at school, or adults (physical attack, threat, bullying, etc.)?"

\section{7-Item Generalized Anxiety Disorder Scale (GAD-7)}

GAD-7 is a self-report scale developed in 2006 for the screening and severity assessment of adults with generalized anxiety disorder. The scale consists of seven items in total, each scored between 0 and 3 points [14]. GAD-7 is widely used in primary healthcare institutions since it can effectively screen for generalized anxiety disorder in a short time, and it was found that severity assessment by GAD-7 has a high correlation with the severity of anxiety symptoms as assessed by clinicians. In a large-scale study conducted with 110000 children and adolescents in Finland, the internal consistency (Cronbach's $\alpha$ value) of GAD-7 was 0.91 . The reliability and validity of the scale in assessing anxiety in this age population were confirmed by a previous study [15]. In Korea, Seo and Park [16] studied the reliability and validity of the Korean translation of GAD-7 in migraine patients. In their study, Cronbach's a was 0.915 , and the sensitivity and specificity were $78.1 \%$ and $74.6 \%$, respectively, which were very close to the levels reported in studies conducted outside of Korea. The internal consistency evaluated in Korean adolescents was an at acceptable level for a large-scale study. In this study, the Korean version of GAD-7 was used, which asked regarding the extent to which students how often have been bothered in their daily lives due to anxiety-related symptoms over the last 2 weeks [17]. In each of the items, students were instructed to check one of the following options: "1) not at all; 2) several days; 3 ) more than half the days; 4) nearly everyday." Based on the total GAD-7 score, anxiety severity was classified as follows: normal if the total score was $0-4$ points, mild anxiety if it was 5-9 points, moderate, $10-14$ points, and severe, 15-21 [14].

\section{Mental health}

The indices defined in the "Statistical Manual for the 16th (2020) Korea Youth Risk Behavior Web-Based Survey” were used for mental health variables [17]. For mental health scales, perceived stress, subjective sleep satisfaction, happiness, loneliness, depressive symptoms, suicidal ideation, suicide planning, and suicide attempts were included. Perceived stress, subjective sleep satisfaction, happiness, and loneliness were assessed with the following items, respectively: "generally, how stressed do you feel?" "do you think that in the last 7 days, you slept long enough to feel rested?" "generally, how happy are you?" and "in the last 12 months, how often have you felt lonely?" Each of the items had five choices, and to conduct logistic regression analysis, the answer choices were categorized into two groups. Depressive symptoms, suicidal ideation, suicide planning, and suicide attempts were assessed with "in the last 12 months, have you ever felt so sad or hopeless for 2 or more weeks in a row that you stopped doing some usual activities?" "in the last 12 months, have you ever thought seriously about suicide?" "in the last 12 months, have you ever made a specific plan to commit suicide?" and "in the last 12 months, have you ever attempted to commit suicide?" respectively. These items were asked by using a dichotomous scale of "yes" or "no."

\section{Health risk behaviors}

Health risk behavior assessment included current smoking status, current drinking status, habitual substance use experience, sexual intercourse experience, and unprotected intercourse experience. Current smoking was defined as smoking regular cigarettes or e-cigarettes for a day or more in the last 30 days and current drinking as consuming a glass or more of alcohol in the last 30 days. Habitual substance use experience was assessed with the item "aside from treatment purposes, have you ever used a drug or substance habitually (e.g., stimulants, antianxiety drugs, butane, glue)?” The response scale was a dichotomous "yes" or "no." Sexual intercourse experience was assessed with the item "have you ever had sexual intercourse?" and students were instructed to choose one out of two choices: "1) have had sexual intercourse" and "2) have not had sexual intercourse." Unprotected intercourse 
experience was assessed using the item "during sexual intercourse, did you use contraceptives?" with the choices being "1) always used contraceptives," "2) almost always used contraceptives," “3) sometimes used contraceptives," and "4) never used contraceptives." The choice of 1) or (2) was categorized as using contraceptives and the others as not using contraceptives.

\section{Statistical analysis}

Participant characteristics were described by analyzing sociodemographics, mental health scales, and health risk behaviors by anxiety severity level. Population percentages were estimated by conducting complex sample analysis. To compare between groups in terms of sociodemographics, mental health scales, and health risk behaviors, continuous data were analyzed by performing an analysis of variance and categorical data by performing the Rao-Scott chi-squared test for significance testing. The sociodemographics that showed between-group difference were included as covariates in the complex-sample logistic regression analysis for adjustment. The odds ratios (OR) and 95\% confidence intervals (CI) of mental health scales and health risk behaviors were computed for each anxiety severity level. Because the sample of the 16th (2020) KYRBS was selected via stratified cluster probability sampling, an analysis based on the assumption of simple random sampling was likely to produce biased results. Hence, to ensure the representativeness of results, complex sampling analysis was performed by generating a complex sample analysis plan file that included stratification, clusters, and weights. Data were analyzed using SPSS for Windows version 25.0 (IBM Corp., Armonk, NY, USA), and the statistical significance level was set at 0.05 in all cases.

\section{RESULTS}

\section{Sociodemographic characteristics}

The 54948 survey respondents' sociodemographic characteristics are presented in Table 1 for each anxiety level determined based on their GAD-7 scores. In total, 36711 (66.6\%) adolescents were classified to have normal anxiety levels, and 12138 (22.2\%), 4134 (7.6\%), and 1965 (3.6\%) were classified to have mild, moderate, and severe anxiety, respectively. In the normal anxiety group, $57.0 \%$ were male and $43.0 \%$ were female, while in the severe anxiety group, $33.8 \%$ were male and $66.2 \%$ were female. In other words, as the anxiety level rose, the proportion of female adolescents increased. Regarding the area of residence estimated for the population, $4.5 \%$ lived in rural areas, $45.1 \%$ in small cities, and $50.3 \%$ in large cities. The area of residence did not show a statistically significant difference according to anxiety severity. With respect to academic achievement, $12.9 \%$ of the normal anxiety group reported their grades as "high," and the proportion was higher

Table 1. General characteristics and academic achievement in each group of anxiety level

\begin{tabular}{|c|c|c|c|c|c|c|}
\hline \multirow[b]{2}{*}{ Variable } & \multirow{2}{*}{$\begin{array}{c}\text { Total } \\
(n=54948)\end{array}$} & \multicolumn{4}{|c|}{ Level of anxiety severity GAD-7 scale score } & \multirow[b]{2}{*}{ p-value } \\
\hline & & $\begin{array}{l}\text { Normal 0-4 } \\
(n=36711)\end{array}$ & $\begin{array}{l}\text { Mild 5-9 } \\
(n=12138)\end{array}$ & $\begin{array}{l}\text { Moderate } 10-14 \\
(n=4134)\end{array}$ & $\begin{array}{c}\text { Severe } 15-21 \\
\quad(n=1965)\end{array}$ & \\
\hline Age (yrs) & $15.19 \pm 0.02$ & $15.09 \pm 0.02$ & $15.35 \pm 0.03$ & $15.38 \pm 0.04$ & $15.49 \pm 0.05$ & $<0.001^{*}$ \\
\hline Family affluence scale & $7.25 \pm 0.02$ & $7.23 \pm 0.02$ & $7.28 \pm 0.03$ & $7.30 \pm 0.03$ & $7.28 \pm 0.05$ & $<0.001^{*}$ \\
\hline \multicolumn{6}{|l|}{ Sex } & $<0.001^{*}$ \\
\hline Male & $28353(51.9)$ & $20880(57.0)$ & $5282(44.0)$ & $1550(38.4)$ & $641(33.8)$ & \\
\hline Female & $26595(48.1)$ & $15831(43.0)$ & $6856(56.0)$ & $2584(61.6)$ & $1324(66.2)$ & \\
\hline \multicolumn{6}{|l|}{ Area of residence } & 0.047 \\
\hline Rural & $3315(4.5)$ & $2191(4.5)$ & $741(4.6)$ & $255(4.3)$ & $128(4.5)$ & \\
\hline Small city & $24198(45.1)$ & $15933(44.7)$ & $5431(45.2)$ & $1953(48.2)$ & $881(45.6)$ & \\
\hline Large city & $27435(50.3)$ & $18587(50.8)$ & $5966(50.2)$ & $1926(47.5)$ & $956(49.8)$ & \\
\hline \multicolumn{6}{|c|}{ History of violence victimization } & $<0.001^{*}$ \\
\hline Yes & $8412(14.9)$ & $5066(13.5)$ & $2087(16.5)$ & 807 (18.9) & $452(22.8)$ & \\
\hline No & $46536(85.1)$ & $31645(86.5)$ & $10051(83.5)$ & $3327(81.1)$ & $1513(77.2)$ & \\
\hline \multicolumn{6}{|l|}{ Academic achievement } & $<0.001^{*}$ \\
\hline Low & $5533(10.0)$ & $3260(8.9)$ & $1290(10.5)$ & 593 (13.9) & $390(19.2)$ & \\
\hline Low-middle & $12684(23.0)$ & $8108(22.0)$ & $3019(24.5)$ & $1048(25.5)$ & $509(26.2)$ & \\
\hline Middle & $16585(30.1)$ & $11404(30.9)$ & $3596(29.7)$ & $1123(27.8)$ & $462(23.9)$ & \\
\hline High-middle & $13410(24.6)$ & $9200(25.3)$ & $2898(24.2)$ & $937(22.6)$ & $375(19.0)$ & \\
\hline High & $6736(12.2)$ & $4739(12.9)$ & $1335(11.1)$ & $433(10.3)$ & $229(11.7)$ & \\
\hline
\end{tabular}

Data are presented as mean \pm standard deviation or $\mathrm{n}(\%)$. Weighted percentages following complex sample analysis. ${ }^{*} \mathrm{p}<0.05$. GAD-7, 7-item Generalized Anxiety Disorder Scale 
than any of the other three groups. The proportion of adolescents responding with "low" was the highest in the severe anxiety group (19.2\%). Regarding the history of violence victimization, the proportion of adolescents having a history was the lowest in the normal anxiety group (13.5\%), and it rose as anxiety levels increased, with the highest in the severe anxi- ety group $(22.8 \%)$.

\section{Comparisons of mental health scales and health risk behaviors according to anxiety level}

The characteristics of mental health scales and health risk behaviors in adolescents according to anxiety level are shown

Table 2. Mental health and health risk behaviors of participants according to anxiety level

\begin{tabular}{|c|c|c|c|c|c|c|}
\hline \multirow[b]{2}{*}{ Variable } & \multirow{2}{*}{$\begin{array}{c}\text { Total } \\
(n=54948)\end{array}$} & \multicolumn{4}{|c|}{ Level of anxiety severity GAD-7 scale score } & \multirow[b]{2}{*}{ p-value } \\
\hline & & $\begin{array}{l}\text { Normal 0-4 } \\
(n=36711)\end{array}$ & $\begin{array}{l}\text { Mild 5-9 } \\
(n=12138)\end{array}$ & $\begin{array}{c}\text { Moderate } 10-14 \\
(n=4134)\end{array}$ & $\begin{array}{c}\text { Severe } 15-21 \\
(n=1965)\end{array}$ & \\
\hline Stress & & & & & & $<0.001^{*}$ \\
\hline High & $18662(34.2)$ & $7141(19.6)$ & $6592(54.4)$ & $3202(77.2)$ & $1727(88.0)$ & \\
\hline Sleep satisfaction & & & & & & $<0.001^{*}$ \\
\hline High & $16824(30.3)$ & $13567(36.5)$ & $2429(20.1)$ & $591(14.5)$ & $237(12.2)$ & \\
\hline Low & $38124(69.7)$ & $23144(63.5)$ & 9709 (79.9) & $3543(85.5)$ & $1728(87.8)$ & \\
\hline Happiness & & & & & & $<0.001^{*}$ \\
\hline High & $35175(63.7)$ & $27677(75.1)$ & 5810 (47.9) & $1300(31.4)$ & $388(19.8)$ & \\
\hline Loneliness & & & & & & $<0.001^{*}$ \\
\hline High & $7766(14.1)$ & $1789(4.8)$ & $2767(22.7)$ & $1917(45.9)$ & $1293(65.0)$ & \\
\hline Low & $47182(85.9)$ & $34922(95.2)$ & 9371 (77.3) & $2217(54.1)$ & $672(35.0)$ & \\
\hline Depressive symptoms & & & & & & $<0.001^{*}$ \\
\hline Yes & $13840(25.2)$ & 4849 (13.3) & $4943(40.5)$ & $2553(61.0)$ & $1495(75.7)$ & \\
\hline No & $41108(74.8)$ & $31862(86.7)$ & $7195(59.5)$ & $1581(39.0)$ & $470(24.3)$ & \\
\hline Suicidal idea & & & & & & $<0.001^{*}$ \\
\hline Yes & 5979 (10.9) & $1491(4.0)$ & $2044(16.7)$ & $1387(33.5)$ & $1057(54.1)$ & \\
\hline No & $48969(89.1)$ & $35220(96.0)$ & $10094(83.3)$ & $2747(66.5)$ & 908 (45.9) & \\
\hline Suicidal attempt & & & & & & $<0.001^{*}$ \\
\hline Yes & $1121(2.0)$ & $248(0.7)$ & $301(2.4)$ & $257(6.1)$ & 315 (15.9) & \\
\hline No & $53827(98.0)$ & $36463(99.3)$ & $11837(97.6)$ & 3877 (93.9) & $1650(84.1)$ & \\
\hline Current smoker & & & & & & $<0.001^{*}$ \\
\hline Yes & $2688(4.8)$ & $1525(4.2)$ & $694(5.6)$ & $280(6.6)$ & $189(9.6)$ & \\
\hline No & $52260(95.2)$ & $35186(95.8)$ & $11444(94.4)$ & 3854 (93.4) & $1776(90.4)$ & \\
\hline Current drinker & & & & & & $<0.001^{*}$ \\
\hline Yes & $5892(10.7)$ & $3288(9.0)$ & $1601(13.0)$ & $635(15.0)$ & $368(18.4)$ & \\
\hline No & $49056(89.3)$ & 33423 (91.0) & $10537(87.0)$ & $3499(85.0)$ & $1597(81.6)$ & \\
\hline \multicolumn{2}{|c|}{ Habitual substance use experience } & & & & & $<0.001^{*}$ \\
\hline Yes & $405(0.8)$ & $148(0.4)$ & $111(1.0)$ & $61(1.4)$ & $85(4.6)$ & \\
\hline No & $54543(99.2)$ & $36563(99.6)$ & $12027(99.0)$ & $4073(98.6)$ & $1880(95.4)$ & \\
\hline \multicolumn{2}{|c|}{ Sexual intercourse experience } & & & & & $<0.001^{*}$ \\
\hline Yes & $2487(4.6)$ & $1376(3.8)$ & $658(5.3)$ & $278(6.7)$ & $175(9.0)$ & \\
\hline No & $52461(95.4)$ & $35335(96.2)$ & $11480(94.7)$ & $3856(93.3)$ & $1790(91.0)$ & \\
\hline Unprotected intercourse & & & & & & $<0.001^{*}$ \\
\hline Yes & $830(1.5)$ & $449(1.3)$ & $222(1.7)$ & $93(2.2)$ & $66(3.5)$ & \\
\hline No & $54118(98.5)$ & $36262(98.7)$ & $11916(98.3)$ & $4041(97.8)$ & $1899(96.5)$ & \\
\hline
\end{tabular}


Table 3. The associations of health risk behavior and mental health with anxiety level

\begin{tabular}{|c|c|c|c|c|}
\hline \multirow{3}{*}{ Variables } & \multicolumn{4}{|c|}{ Level of anxiety severity GAD-7 scale score } \\
\hline & \multirow{2}{*}{ Normal $0-4$} & Mild 5-9 & Moderate 10-14 & Severe $15-21$ \\
\hline & & OR $(95 \% \mathrm{Cl})$ & OR $(95 \% \mathrm{Cl})$ & OR $(95 \% \mathrm{Cl})$ \\
\hline \multicolumn{5}{|l|}{ Mental health } \\
\hline Stress & Reference & $4.63(4.43-4.84)$ & $12.857(11.88-13.92)$ & $26.39(23.05-30.21)$ \\
\hline Sleep satisfaction & Reference & $0.47(0.45-0.50)$ & $0.33(0.30-0.36)$ & $0.28(0.24-0.32)$ \\
\hline Happiness & Reference & $0.31(3.00-3.33)$ & $0.16(0.15-0.17)$ & $0.09(0.08-0.10)$ \\
\hline Loneliness & Reference & $5.48(5.14-5.85)$ & $15.36(14.21-16.60)$ & $32.08(28.63-35.95)$ \\
\hline Depressive symptoms & Reference & $4.16(3.98-4.36)$ & $9.23(8.61-9.86)$ & $17.61(15.73-19.72)$ \\
\hline Suicidal idea & Reference & $4.53(4.21-4.86)$ & $11.02(10.10-12.02)$ & $24.53(21.87-27.50)$ \\
\hline Suicidal attempt & Reference & $3.56(3.02-4.20)$ & $8.70(7.23-10.48)$ & $22.87(19.10-27.37)$ \\
\hline \multicolumn{5}{|l|}{ Health risk behaviors } \\
\hline Current smoker & Reference & $1.33(1.20-1.48)$ & $1.55(1.34-1.80)$ & $2.08(1.72-2.50)$ \\
\hline Current drinker & Reference & $1.43(1.34-1.52)$ & $1.65(1.50-1.82)$ & $1.91(1.67-2.19)$ \\
\hline Habitual substance use experience & Reference & $2.40(1.89-3.04)$ & $3.47(2.56-4.71)$ & $10.89(8.22-14.42)$ \\
\hline Sexual intercourse experience & Reference & $1.35(1.22-1.48)$ & $1.74(1.52-1.98)$ & $2.10(1.76-2.51)$ \\
\hline Unprotected intercourse & Reference & $1.36(1.17-1.63)$ & $1.69(1.35-2.12)$ & $2.21(1.67-2.92)$ \\
\hline
\end{tabular}

in Table 2. Between-group differences were statistically significant in all mental health scales and health risk behaviors.

Regarding the mental health scales by anxiety level, the severe anxiety group showed the highest proportions of perceived stress (88.0\%), loneliness $(65.0 \%)$, depressive symptoms (75.7\%), suicidal ideation (54.1\%), and suicide attempts (15.9\%). On the other hand, the normal anxiety group showed the highest proportions of subjective sleep satisfaction (36.5\%) and happiness (75.1\%).

With regard to health risk behaviors by anxiety level, the proportions of current smokers were $4.2 \%$ in the normal anxiety group, $5.6 \%$ in the mild anxiety group, $6.6 \%$ in the moderate anxiety group, and $9.6 \%$ in the severe anxiety group. The proportions of current drinkers were 9.0\%, 13.0\%, 15.0\%, and $18.4 \%$ in the normal, mild, moderate, and severe anxiety groups, respectively. The respective proportions of adolescents with habitual substance use experience were $0.4 \%, 1.0 \%$, $1.4 \%$, and $4.6 \%$ in the groups with increasing anxiety severity from normal to severe. The respective proportions of adolescents with sexual intercourse experience were 3.8\%, 5.3\%, $6.7 \%$, and $9.0 \%$ in the groups with increasing anxiety severity from normal to severe. Similarly, the proportions of adolescents with unprotected intercourse experience were $1.3 \%$, $1.7 \%, 2.2 \%$, and $3.5 \%$ in the groups with increasing anxiety severity from normal to severe.

Table 3 shows the ORs for mental health scales and health risk behaviors that were adjusted for the sociodemographic characteristics, showing significant between-group differences (age, family affluence, sex, academic achievement, and a history of violence victimization). The ORs for each mental health scale in the severe anxiety group were as follows: perceived stress - 26.386 (95\% CI: 23.05-30.21), subjective sleep satisfaction - 0.28 (95\% CI: 0.24-0.32), happiness - 0.09 (95\% CI: 0.08-0.10), loneliness - 32.08 (95\% CI: 28.63-35.95), depressive symptoms - 17.61 (95\% CI: 15.73-19.72), suicidal ideation - 24.53 (95\% CI: 21.87-27.50), and suicide attempts 22.87 (95\% CI: 19.10-27.37). With the normal anxiety group as the reference, the ORs for health risk behaviors (i.e., current smoker, currently drinking, habitual substance use, sexual intercourse, and unprotected intercourse) increased as the anxiety level increased from mild to moderate to severe. The ORs for health risk behaviors in the severe anxiety group were as follows: current smoking - 2.08 (95\% CI: 1.72-2.50), current drinking - 1.91 (95\% CI: 1.67-2.19), habitual substance use - 10.89 (95\% CI: 8.22-14.42), sexual intercourse - 2.10 (95\% CI: 1.76-2.51), and unprotected intercourse - 2.21 (95\% CI: 1.67-2.92).

\section{DISCUSSION}

In this study, the associations between anxiety severity and sociodemographic characteristics, mental health, and health risk behaviors in adolescents were examined using a large sample. It is of significance in that this is the first study in Korea to utilize a large-scale sample to investigate the association between anxiety severity and other psychopathologies in adolescence.

Regarding sociodemographic characteristics, the propor- 
tion of female adolescents increased as anxiety severity increased. Being a female is a risk factor for anxiety disorder, and the sex ratio of anxiety disorder in adolescence was reported to be $1: 2$ to $1: 3$ [18]. In this study, the sex ratio in the severe anxiety group was also approximately 1:2, a level similar to previous study findings. In most epidemiological studies, participants with a low educational attainment level had a higher anxiety level than those with higher educational attainment levels [18]. However, the temporal relationship between the educational attainment level and anxiety has not yet been clearly demonstrated. In this study, the proportion of adolescents who reported their grades to be low increased as the anxiety level increased, but the proportion of those who reported their grades to be high did not show a consistently increasing or decreasing trend with increasing anxiety severity. Academic achievement was assessed using a self-reported question, and the finding may have been limited by the lack of an objective assessment of academic achievement. In a previous study on the history of violence victimization, early experiences of loneliness and social anxiety were the predictors of violence victimization [19]. In this study, the proportion of adolescents with a history of violence victimization was high in the severe anxiety group. However, causal relationships, namely, whether a history of violence victimization was an early negative experience that influenced anxiety level or whether a high anxiety level had an impact on violence victimization, was not examined.

The ORs for mental health scales increased more sharply with increasing anxiety level than did the ORs for health risk behaviors. In the severe anxiety group, loneliness showed the strongest association (OR: 32.08), followed by stress (OR: 26.39), suicidal ideation (OR: 24.53), suicide attempts (OR: 22.87), and depressive symptoms (OR: 17.61). This group was also associated with decreased subjective sleep satisfaction (OR: 0.28) and happiness (OR: 0.09). In particular, the experience of loneliness (OR: 32.08) had the strongest association with anxiety severity. The proportion of adolescents with an experience of loneliness was $4.8 \%$ in the normal anxiety group, whereas the corresponding number was $65.0 \%$ in the severe anxiety group. This finding is in line with the previous studies which demonstrated that loneliness and anxiety are strongly associated with each other [20]. Some studies reported that loneliness is a mediator of social anxiety and suicidal ideation [21]. In summary, these findings provide a basis for the need to assess loneliness in parallel when evaluating adolescents experiencing anxiety. A review of the literature on the relationship between anxiety and stress showed that there is a functional change in the hypothalamic-pituitary-adrenal axis and the autonomic nervous system in children with anxiety disorder and that the change is similar to what happens due to long-term exposure to stress [22]. As a hypothesis regarding the cause of the functional change, an animal model, which showed that stress is involved in the expression of glucocorticoid receptors in the hippocampus, has been put forward [23]. Based on the study finding that the proportion of perceived stress in the severe anxiety group was very high (88.0\%), it can be speculated that anxiety and stress may share a common factor.

It has been consistently found that anxiety is significantly associated with suicidal ideation and attempts [24]. In this study, both suicidal ideation and suicide attempts also increased as anxiety levels increased. In the severe anxiety group, the association with anxiety severity was stronger for suicidal ideation (OR: 24.53) and suicide attempts (OR: 22.87) than for depressive symptoms (OR: 17.61). The increase in the ORs of depressive symptoms according to increasing levels of anxiety severity, that is, high levels of co-existence of anxiety and depression in childhood and adolescence, may be due to the presence of common biological factors [25]. On the other hand, the co-occurrence can also be explained by overlapping symptoms in the diagnoses of anxiety and depressive disorders. For example, the symptoms of fatigue, sleep disorder, and attention disorder, plus oversensitivity in the case of children and adolescents, are included in both diagnostic criteria for generalized anxiety disorder and major depressive disorder [25]. Not only is GAD-7 useful in assessing the severity of anxiety symptoms in adolescence, but it also reflects the severity of depression symptoms [14], which may be reflective of the presence of common factors in depression and anxiety.

Sleep-related problems are known to be associated with the severity of anxiety symptoms [26]. Although sleep-related problems are widely prevalent in adolescents with anxiety disorder, currently, clear evidence is absent regarding the temporal relationship. In this study, the severer the anxiety symptoms, the lower the subjective sleep satisfaction. Additional research should be performed to investigate the causality regarding subjective sleep satisfaction and the association between anxiety and sleep-related problems, an issue not examined in this study. A previous study on anxiety and happiness found low-level happiness in patients with major depressive disorder and with social anxiety disorder [27]. Happiness is defined as positive subjective experiences, and it has been shown that the happier the person is, the lower their anxiety and tension in stressful situations [28]. So far, the link between anxiety and happiness has not been sufficiently studied. In this study, the increase in anxiety levels as assessed via GAD-7 was associated with a decrease in happiness, and a follow-up study should be conducted to identify the causality between anxiety symptoms and happiness. 
Regarding health risk behaviors, as anxiety levels increased, the ORs increased for current smokers, current drinkers, habitual substance use, sexual intercourse, and unprotected intercourse. Various studies demonstrated that smoking is associated with increased anxiety. It has been argued that early exposure to smoking tends to reinforce later anxiety through a variety of mechanisms, such as antioxidants, oxidative stress, and brain injury, and that smoking has the effect of mood elevation in depressive adolescents [29]. It is plausible for both of these factors to be involved, given the study finding that the ORs of current smokers increased with increasing anxiety severity in adolescents. However, this cross-sectional study was limited in confirming the temporal relationship.

According to a meta-analysis study published in 2019 that examined 51 articles on drinking [30], the relationships of anxiety to drinking frequency and volume and binge drinking were not clear, and the evidence for the association between generalized anxiety disorder and alcohol use was sparse, though there was some evidence for the associations between alcohol use and other types of anxiety disorder and physiological anxiety. In summarizing existing study findings, we concluded that it is difficult to determine an association between anxiety in adolescence and later alcohol use, but suggested that the lack of evidence may have been due to between-study differences with respect to the control of confounding variables and study design. So far, numerous observational studies have reported that anxiety is associated with drinking [31], and that the association between the two is explained by one of the following hypotheses: 1) anxious persons drink to assuage physiological and emotional reactions, and 2) repeated drinking induces anxiety. Similarly, in this study, the ORs of current drinkers steadily increased as anxiety severity increased. In a nationwide epidemiological study conducted with 40000 adults aged $18-44$ y in the US, anxiety disorder was associated with alcohol use and substance use disorders, with a stronger association with substance dependence (OR: 6.0) than with alcohol dependence (OR:3.0) [32]. In this study, as anxiety severity increased, its associations with current drinkers and habitual substance use both tended to increase as well, but the study was limited in that drinking and substance use could not be compared with each other due to inconsistencies in the survey items.

It has been reported that health risk behaviors in adolescence may show the characteristics of manifesting various problem behaviors in combination, and that risky sexual behavior in adolescence is associated with problem behaviors like substance abuse, drinking, and smoking [33]. Increased anxiety levels are associated with substance use, and could also be associated with an increased possibility of the development of risky sexual behavior. In this study, as anxiety lev- els increased, the ORs of sexual intercourse, unprotected intercourse, and other risk behaviors, such as smoking and drinking, increased at the same time.

The limitations of the study were as follows. First, the KYRBS has limitations as a self-report survey filled out by adolescents. The survey was performed online during class at school; thus, it may have been plausible that the behaviors that adolescents did not want to reveal (such as sexual behavior, smoking, drinking, violence victimization, and suicide) were underreported because respondents feared the consequences of disclosing their behavioral characteristics. As the information on academic achievement was obtained via the respondents' personal opinions, objectivity in the data could have been lacking. In the responses to items asking about experiences in the last 12 months, recall bias may have occurred. Second, this study was cross-sectional, and causal or temporal relationships between anxiety and other scales could not be clearly explained. Third, of the items pertaining to mental health, the subjective feeling of happiness and perceived stress were not accompanied with objectified information, Thus, it is possible that the responses did not accurately reflect the adolescents' states. For instance, the survey item regarding depressive symptoms did not mean to assess clinical major depressive disorder. Fourth, in this study, anxiety level was classified using GAD-7 scores. While GAD-7 was developed as a scale to evaluate the severity of generalized anxiety disorder, it may have been difficult to assess all anxiety symptoms using GAD-7. However, based on the studies conducted thus far, GAD-7 seems appropriate in assessing anxiety disorders other than generalized anxiety disorder. The scale has been shown to be useful in assessing panic disorder (sensitivity, 74\%; specificity, $81 \%$ ), social anxiety disorder (sensitivity, $72 \%$; specificity, $80 \%$ ), and post-traumatic stress disorder (sensitivity, 66\%; specificity, 81\%) [34]. Hence, in consideration of the advantage that anxiety symptoms can be assessed with simple items in a short time, it is believed that GAD-7 can be effectively used to assess the level of anxiety in a large sample.

\section{CONCLUSION}

The study found that an increased level of anxiety in adolescence was associated with high scores in a variety of mental health scales and with health risk behaviors. Because anxiety symptoms during adolescence can be an early manifestation of various psychopathologies, adolescents with high anxiety levels are vulnerable to other anxiety disorders and other mental disorders. To prevent this, adolescents in the community who show severe anxiety symptoms should be screened early and be specifically managed and intervened with respect 
to anxiety symptoms, health risk behaviors, and mental disorders that might occur at a later point in time. In the future, large-scale longitudinal research should be performed to identify causal relationships between anxiety levels and other psychopathologies and health risk behaviors in adolescents.

\section{Availability of Data and Material}

The raw dataset analyzed during the current study are available at http://www.kdca.go.kr/yhs/

\section{Conflicts of Interest}

The authors have no potential conflicts of interest to disclose

\section{Author Contributions}

Conceptualization: all authors. Data curation: Kyung Soo Woo. Investigation: Kyung Soo Woo. Project administration: Yoonmi Ji, Hye Jeong Lee. Supervision: Tae Young Choi. Validation: Yoonmi Ji, Hye Jeong Lee. Writing_original draft: Kyung Soo Woo. Writing_review \& editing: Yoonmi Ji, Hye Jeong Lee, Tae Young Choi.

\section{ORCID iDs}

Kyung Soo Woo https://orcid.org/0000-0001-5295-4787

Yoonmi Ji https://orcid.org/0000-0002-9964-197X

Hye Jeong Lee https://orcid.org/0000-0003-1615-527X

Tae Young Choi https://orcid.org/0000-0003-2677-9297

\section{Funding Statement}

None

\section{REFERENCES}

1) American Psychiatric Association. Diagnostic and statistical manual of mental disorders: DSM-5. 5th ed. Arlington, VA: American Psychiatric Association;2013.

2) Shear MK, Bjelland I, Beesdo K, Gloster AT, Wittchen HU. Supplementary dimensional assessment in anxiety disorders. Int J Methods Psychiatr Res 2007;16 Suppl 1:S52- S64.

3) Merikangas KR, He JP, Burstein M, Swanson SA, Avenevoli S, Cui L, et al. Lifetime prevalence of mental disorders in U.S. adolescents: results from the National Comorbidity Survey Replication-Adolescent Supplement (NCS-A). J Am Acad Child Adolesc Psychiatry 2010;49:980-989.

4) Woodward LJ, Fergusson DM. Life course outcomes of young people with anxiety disorders in adolescence. J Am Acad Child Adolesc Psychiatry 2001;40:1086-1093.

5) Wittchen HU, Lieb R, Pfister H, Schuster P. The waxing and waning of mental disorders: evaluating the stability of syndromes of mental disorders in the population. Compr Psychiatry 2000;41:122132.

6) Kessler RC, Crum RM, Warner LA, Nelson CB, Schulenberg J, Anthony JC. Lifetime co-occurrence of DSM-III-R alcohol abuse and dependence with other psychiatric disorders in the National Comorbidity Survey. Arch Gen Psychiatry 1997;54:313-321.

7) Gullone E. Developmental psychopathology and normal fear. Behav Change 1996;13:143-155.

8) Merikangas KR, He JP, Burstein M, Swendsen J, Avenevoli S, Case B, et al. Service utilization for lifetime mental disorders in U.S. adolescents: results of the National Comorbidity Survey-Adolescent Supplement (NCS-A). J Am Acad Child Adolesc Psychiatry 2011;50:32-45.

9) Igra V, Irwin CE. Theories of adolescent risk-taking behavior. In: DiClemente RJ, Hansen WB, Ponton LE, editors. Handbook of ad- olescent health risk behavior. Boston: Springer;1996. p.35-51.

10) Brener ND, Collins JL. Co-occurrence of health-risk behaviors among adolescents in the United States. J Adolesc Health 1998;22: 209-213.

11) Flory K, Lynam D, Milich R, Leukefeld C, Clayton R. Early adolescent through young adult alcohol and marijuana use trajectories: early predictors, young adult outcomes, and predictive utility. Dev Psychopathol 2004;16:193-213.

12) Pailing AN, Reniers RLEP. Depressive and socially anxious symptoms, psychosocial maturity, and risk perception: associations with risk-taking behaviour. PloS one 2018;13:e0202423.

13) Andersen A, Krølner R, Currie C, Dallago L, Due P, Richter M, et al. High agreement on family affluence between children's and parents' reports: international study of 11-year-old children. J Epidemiol Community Health 2008;62:1092-1094.

14) Spitzer RL, Kroenke K, Williams JB, Löwe B. A brief measure for assessing generalized anxiety disorder: the GAD-7. Arch Intern Med 2006;166:1092-1097.

15) Tiirikainen K, Haravuori H, Ranta K, Kaltiala-Heino R, Marttunen M. Psychometric properties of the 7-item Generalized Anxiety Disorder Scale (GAD-7) in a large representative sample of Finnish adolescents. Psychiatry Res 2019;272:30-35.

16) Seo JG, Park SP. Validation of the Generalized Anxiety Disorder-7 (GAD-7) and GAD-2 in patients with migraine. J Headache Pain 2015;16:97.

17) Korea Disease Control and Prevention Agency. The 16th reports on the Korea youth risk behavior web-based survey (2020). Cheongju: Korea Disease Control and Prevention Agency;2021 [cited 2021 Jun 1]. Available from: http://www.kdca.go.kr/yhs/yhshmpg/ result/yhsresult/statsBookList.do.

18) Wittchen HU, Nelson CB, Lachner G. Prevalence of mental disorders and psychosocial impairments in adolescents and young adults. Psychol Med 1998;28:109-126.

19) Acquah EO, Topalli PZ, Wilson ML, Junttila N, Niemi PM. Adolescent loneliness and social anxiety as predictors of bullying victimisation. Int J Adolesc Youth 2016;21:320-331.

20) Lim MH, Rodebaugh TL, Zyphur MJ, Gleeson JF. Loneliness over time: the crucial role of social anxiety. J Abnorm Psychol 2016;125: 620-630.

21) Gallagher M, Prinstein MJ, Simon V, Spirito A. Social anxiety symptoms and suicidal ideation in a clinical sample of early adolescents: examining loneliness and social support as longitudinal mediators. J Abnorm Child Psychol 2014;42:871-883.

22) Dieleman GC, Huizink AC, Tulen JH, Utens EM, Creemers HE, van der Ende J, et al. Alterations in HPA-axis and autonomic nervous system functioning in childhood anxiety disorders point to a chronic stress hypothesis. Psychoneuroendocrinology 2015;51:135150 .

23) Tsoory M, Cohen H, Richter-Levin G. Juvenile stress induces a predisposition to either anxiety or depressive-like symptoms following stress in adulthood. Eur Neuropsychopharmacol 2007;17: 245-256.

24) Bentley KH, Franklin JC, Ribeiro JD, Kleiman EM, Fox KR, Nock MK. Anxiety and its disorders as risk factors for suicidal thoughts and behaviors: a meta-analytic review. Clin Psychol Rev 2016;43: 30-46.

25) Garber J, Weersing VR. Comorbidity of anxiety and depression in youth: implications for treatment and prevention. Clin Psychol (New York) 2010;17:293-306.

26) Chase RM, Pincus DB. Sleep-related problems in children and adolescents with anxiety disorders. Behav Sleep Med 2011;9:224-236.

27) Spinhoven P, Elzinga BM, Giltay E, Penninx BW. Anxious or depressed and still happy? PLoS One 2015;10:e139912.

28) Horiuchi S, Tsuda A, Toyoshima N, Aoki S, Sakano Y. Association of increased levels of happiness with reduced levels of tension and 
anxiety after mental stress testing in Japanese college students. Psychology (Irvine) 2013;4:682-687.

29) Moylan S, Jacka FN, Pasco JA, Berk M. How cigarette smoking may increase the risk of anxiety symptoms and anxiety disorders: a critical review of biological pathways. Brain Behav 2013;3:302-326.

30) Dyer ML, Easey KE, Heron J, Hickman M, Munafò MR. Associations of child and adolescent anxiety with later alcohol use and disorders: a systematic review and meta-analysis of prospective cohort studies. Addiction 2019;114:968-982.

31) Glantz MD, Anthony JC, Berglund PA, Degenhardt L, Dierker L,
Kalaydjian A, et al. Mental disorders as risk factors for later substance dependence: estimates of optimal prevention and treatment benefits. Psychol Med 2009;39:1365-1377.

32) Smith JP, Book SW. Anxiety and substance use disorders: a review. Psychiatr Times 2008;25:19-23.

33) Baskin-Sommers A, Sommers I. The co-occurrence of substance use and high-risk behaviors. J Adolesc Health 2006;38:609-611.

34) Kroenke K, Spitzer RL, Williams JB, Monahan PO, Löwe B. Anxiety disorders in primary care: prevalence, impairment, comorbidity, and detection. Ann Intern Med 2007;146:317-325. 